\title{
Analysis of Optical Radiation Generated by Partial Discharges on Insulators
}

\author{
P. FrąCZ*, T. BOCZAR AND S. BORUCKI \\ Institute of Power Engineering and Renewable Energy, Faculty of Electrical Engineering, Automatic Control \\ and Computer Science, Opole University of Technology, Prószkowska 76, 45-758 Opole, Poland
}

\begin{abstract}
Results of measurements of optical radiation emitted by partial and complete electrical discharges occurring on a bushing insulator model placed in air are presented in the article. Registration was performed using a spectrophotometer, enabling for measurement of optical radiation in the range from 270 to $1700 \mathrm{~nm}$. Detailed characteristics of spectrophotometer are presented in our previous paper. The primary objective of the laboratory tests was to evaluate the impact of supply voltage changes in the range from 0 to $0.99 U_{\mathrm{b}}$ (breakdown voltage) on the intensity and spectral character of the optical radiation emitted by partial discharges generated on the porcelain insulation surface of the bushing insulator. In the scope of studies analyzes of the effects of voltage changes on the obtained spectral waveforms were performed, whereby voltage increase and reduction was examined separately. Additionally, measurements and analyzes carried out included impact estimation of the time period as the voltage was applied to the high-voltage electrodes on the course of optical phenomena related to the generation of partial discharges.
\end{abstract}

DOI: 10.12693/APhysPolA.125.1360

PACS: $79.60 . \mathrm{Bm}, 42.79 . \mathrm{Pw}, 52.80 . \mathrm{Hc}$

\section{Introduction}

One of the basic problems of insulation systems used in electrical equipment and high voltage transmission lines is the process of aging, which progresses during their service, and deterioration of insulating properties caused by this process. Reduction in aging resistance is caused by a number of external factors, among which atmospheric, environmental, and voltage exposures can be mentioned. Important aging factors that can be found during their exploitation, include: UV radiation, the presence of ozone and oxides of nitrogen, temperature fluctuations, rain (including acid rain), depositions of rime or dirt, and partial discharges (PDs) [1, 2].

At present, the main objective of the diagnostic research is answering, as precise as possible, the questions on the manner, time, and duration of the insulation element deterioration process, and additionally on conditions under which the total damage will occur.

Currently used diagnostic techniques usually allow monitoring of selected parameters, criterion indices of which inform about the possibility of breakdowns and permanent damage of the examined unit. However, in recent years, rapid development of various techniques for monitoring, analysis and assessment of the operation state of electrical equipment, being now an integral element of the energy power production, transmission and distribution processes can be noticed [3]. Contaminants that accumulate on the surface of high voltage insulators reduce their insulating properties. Presence of pollution,

*corresponding author; e-mail: pfracz@gmail.com as well as high humidity cause formation of the conductive paths, which are the source of PDs [4-10]. Number, amplitude and intensity of PDs which can be generated in high voltage power devices are the critical values when assessing and determining the quality of insulation. Their presence can have a direct impact on the stability and proper operation of the power system because they can contribute to a failure and in extreme cases can cause power supply breakdowns. Hence, there is continuous development of various techniques for detection, location and measurement of the PD intensity [11-14].

Application of new methods for measuring signals, and most of all, for processing recorded data and analysing results, allows performing more accurate, detailed, and thus, more objective assessment of the insulation condition being examined. Data registration can be done on-line (such parameters as: voltage, current, loads, and temperature measured in the normal operation of the device) or off-line (suitable measuring conditions are created for different cases of device exposure, while tests are carried out mostly in the laboratory conditions). Typical off-line methods for diagnostics of PD generation are measures of: loss factor, insulation resistance, as well as polarisation and depolarisation currents [15-17].

In addition to the conventional methods for PD measurement, which are defined in IEC 60270 [18] (electrical methods, HF/VHF, UHF), nondestructive alternative methods are most often used for diagnosis of insulation systems [19-21]. The latter group includes optical, mechanical (acoustic and optoacoustic) and chemical methods. Electrical method is mostly used for detection and quantitative assessment of discharges. However, acoustic method also allows for location of areas, where PDs occur. In practical diagnostic applications, there are also 
applied methods for radio interference voltage measurement, thermography and visual observations. The phenomenon of temperature raise in the area where PDs occur in gas centres were described, inter alia, in [4, 22]. In [1], the authors state that the best effect to determine the condition of insulation can be obtained by using several methods combined and the pattern recognition techniques. Monitoring and determination of dependences between electric field and properties of insulating material is called dielectric diagnostic method. By its use, specific parameters for the insulation system and the electric power device under test are measured, which are: capacity, loss factor, and insulation resistance, from which it is possible to determine changes occurring in the unit under diagnostics.

Objectives of detection are structural changes, humidity, presence of pollutions and PDs. Construction of measurement system largely depends on the value of parameters describing the phenomenon of $\mathrm{PD}$ generation, and in particular, on the frequency spectra, the shape of waveforms, and time-frequency dependence. Ignorance of these descriptors can lead to significant measurement errors. For example: in gas insulated systems (GIS) systems, the generated PDs with raise time less than ns, emit electromagnetic waves with frequencies in the $\mathrm{GHz}$ frequency range. Therefore, systems used in the diagnostics allow measurements in the ultra high frequency (UHF) range, at frequency range of at least up to $2 \mathrm{GHz}$.

In the case of polymeric insulation, frequencies of the emitted electromagnetic waves are in the range up to $100 \mathrm{MHz}$, and therefore, the applied measurement systems allow performing measurements in the very high frequency (VHF) range [10]. Possibility of measuring PDs that occur in high voltage devices and insulations as applicable to diagnostics, and examples of measurement systems that use various diagnostic methods were described, among others, in [1-8]. Among alternative methods for measuring PDs can be mentioned optical spectrophotometry, which is based on detection and measurement of optical radiation being emitted due to a series of processes related with ionisation, excitation and recombination that occur during PD generation [10-14]. Subject matter of this paper is to determine feasibility and indicate the scope of the optical spectrophotometry methods for PD detection, which can occur on the surface of insulators.

\section{Insulator model system selection where PDs occur}

Bushing insulator is a structure-type system that allows conducting one or few wires through walls or partitions of the transformer, or in power distribution stations through tanks and through grounded transformer covers, capacitors, generators, transformers and switches, and insulating them from this partition. At the same time, the fitting (flange or clamping device) to partition is part of the bushing insulator. In the most general manner, bushing insulators can be divided into station and apparatus insulator units. Depending upon the use, operating conditions and operating functions, they can be distinguished for indoor, outdoor and air-to-oil application [22].

Bushing insulator is a classic example of diagonally layered system. Voltage distribution in such an insulation system depends primarily on special capacity and capacitance of a dielectric material sandwiched between electrodes [13-17]. Based on empirical dependences, which can be determined by flashover voltage value and starting voltage of sliding discharges for two most commonly used flat-type and cylindrical bushing systems, bushing equivalent circuit, distributions of electric field lines within a solid material and in air near bushing insulation surface, and also interactions of normal and tangential components were presented, among others, in [8, 22]. While, a detailed description of the phenomena of electrical discharge generation in air, breakdown mechanisms of solid insulating materials, the surface resistance of insulation systems, flashover voltage on polluted traction insulators and related with it permanent degradation of solid dielectric surface, as well as description of flashover phenomena in a bushing-type system, assessment of effect of waveforms of flashover voltage, description of exposure of electrical, mechanical, thermal and pollution origin that can occur under operating conditions, aging processes in insulators, and failure frequency caused by them were presented, inter alia, in $[4,14,22]$.

For PD generation on the surface of bushing insulator, two model systems were used; their schematic representation and real view are presented in Fig. 1a (model I) and $\mathrm{b}$ (model II). The models applied were made of porcelain, and additionally, in the case of model I, into eight of openings arranged within the length of the insulating bush, brand new transformer oil was introduced. Each of the symmetrically arranged openings had a diameter of $0.7 \mathrm{~cm}$. Furthermore, the models used varied in dimensions, which is in diameter $(5 \mathrm{~cm}$ and $6 \mathrm{~cm}-$ models I and II, respectively) and height (34 cm and $44 \mathrm{~cm}$ models I and II, respectively). For the purpose of modeling the bushing insulators (models I and II), inlays with diameters ranging from 4.9 to $9.9 \mathrm{~mm}$ were put into the openings made inside the porcelain bushings.

The examined models of insulators were powered by testing circuit that allows stepless regulation of the applied voltage in the range from 0 to $60 \mathrm{kV}$. Power supply system was used with scheme, which consisted of an automatic control and voltage measurement circuit, a control panel and a testing transformer.

\section{Characteristics of optical radiation measurement system}

To measure optical radiation emitted by PDs generated in bushing insulator modeling system, a spectrophotometer was used with optical transducer placed in a holder made for this purpose, attached to a tripod. The tripod, which was used allows for adjustments of height, 


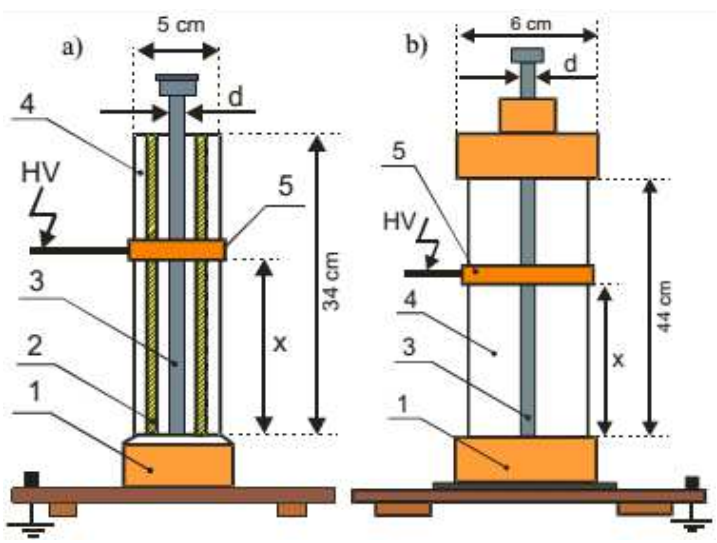

Fig. 1. Schematic diagram of the system modeling bushing ( $\mathrm{a}$ - model I, b - model II) insulators, where: 1 - grounded fitting, 2 - transformer oil, 3 - metal inlay, 4 - ceramic insulation, 5 - movable metal clamp.

tilt angle and distance between the measuring header and examined insulator. During the research tests, AVS-USB2000 spectrometer by OceanOptics, Inc. was used, the main element of which is a multi-stage diffraction grating that allows the analysis of optical spectra in the range $270-1700 \mathrm{~nm}$ with resolution of about $0.5 \mathrm{~nm}$. Recorded signal is distributed to the grid through the fiber. After dispersion, the radiation falls on the CCD (Sony ILX511).

Detailed characteristics of the used spectrophotometer were presented in $[14,22]$. The spectrophotometer applied was connected with a PC through a serial port. For triggering measurements and initial preview of the obtained spectral dependences, standard software offered by OceanOptics, Inc. was used. Whereas, for archiving purposes and further processing of the recorded measurement data, numerical procedures implemented in the Matlab programming environment were used.

Measurements of optical spectra obtained using the spectrophotometer are burdened with a relatively high degree of uncertainty, particularly this applies to low voltages (less than $0.5 U_{\mathrm{b}}$ ) and to large distances between the input of fiber optic measuring head of the spectrophotometer, and the object of PD generation (over $20 \mathrm{~cm}$ ). Therefore, in order to assess the obtained spectral structures, software for automatic detection of linear spectral components, using a set of Gaussian function was developed. The written procedures use the Nelder-Mead method for searching an optimal set of approximating functions that ensure minimal deviation values. The final result is the sum of spectral components in the form of dependences expressed by Eq. (1):

$$
I(f)=\sum_{i=1}^{N} A_{i} e^{-\left(\frac{f-f_{i}}{d}\right)},
$$

where $A_{i}$ - amplitude of the constituent fringe with the length of $d=1.8$ - adopted width of a single fringe, $N$ - wavelength of optical radiation.
Once, the local extremes of the function (1) were found for selected dominant wavelengths, for which the maximum values of optical radiation intensity were designated, its changes were designated as a function of voltage. In order to determine functions modeling dependences of the registered radiation intensity for particular fringes as a function of input voltage used in a series of models. The most representative models were: single exponential model presented by Eq. (2) and two-parameter power model expressed by the relation (3):

$$
I(U)=\mathrm{e}^{A U},
$$

where $A$ - constant coefficient in the model, $U$ - value of the supply voltage.

$$
I(U)=A U^{B},
$$

where $A, B$ - constant coefficients in the model, $U$ value of the supply voltage.

\section{Results of measurements and analyses of optical signals registered using spectrophotometer}

The scope of measurements performed on optical radiation emitted by PD generated on bushing insulator modelling system covered following cases:

a) assessment of the impact of intensity and the nature of waveforms in the spectra of optical radiation was performed, when changing the supply voltage values within the range of 0 to $0.99 U_{\mathrm{b}}$ (breakdown voltage). Moreover, a comparative analysis of the calculated spectra, obtained by increasing and decreasing the supply voltage was performed during the research tests.

b) assessment of the effect of PD generation time in the range from 0 to $25 \mathrm{~min}$ on the obtained waveforms of the emitted optical radiation spectra was performed. At the same time, measurements were conducted at the same supply voltage equal to $0.99 U_{\mathrm{b}}$.

To perform comparative analyses of the obtained results, the research, scope of which was defined in points (a) and (b), was performed at the same distance $x=12 \mathrm{~cm}$ between the aluminium clip (label-5 in Fig. 1a and b) to which high potential power supply and grounded ferrule (label-1 in Fig. 1a and b) were connected. Recording optical radiation emitted by PDs, generated in bushing insulator modeling systems (model I and II) were performed for aluminium inlay with a diameter of $9.9 \mathrm{~mm}$ (label-3 in Fig. 1a and b). Furthermore, comparison measurements were performed for inlays with the diameter of: $4.9,5.9,6.9,7.9$, and $8.9 \mathrm{~mm}$.

Based on the obtained results, it was indicated that change in the diameter does not affect the shape of waveforms of the designated optical radiation spectra in both bushing insulator models. Therefore, this paper presents results obtained only for one diameter of the inlay. During each series of measurements, the distance between 
the spectrophotometer measurement head and the place of PD generation was constant and equal to $3 \mathrm{~cm}$. Measurements were performed in a darkened room, without access to any additional light of ratio sources, of the laboratory for electric power transformer insulation at the Institute of Electric Power Engineering and Renewable Energy of the Technical University of Opole.

Figure 2 presents spectra of optical radiation emitted by PD generated in bushing insulator models (model I and II - Fig. 2), obtained when increasing (a1) and decreasing (a2) the supply voltage value, respectively. Spectra, shown in Fig. 2, were designated for the whole range of wavelengths of optical radiation within the range of 270-1700 nm, registered using spectrophotometer.

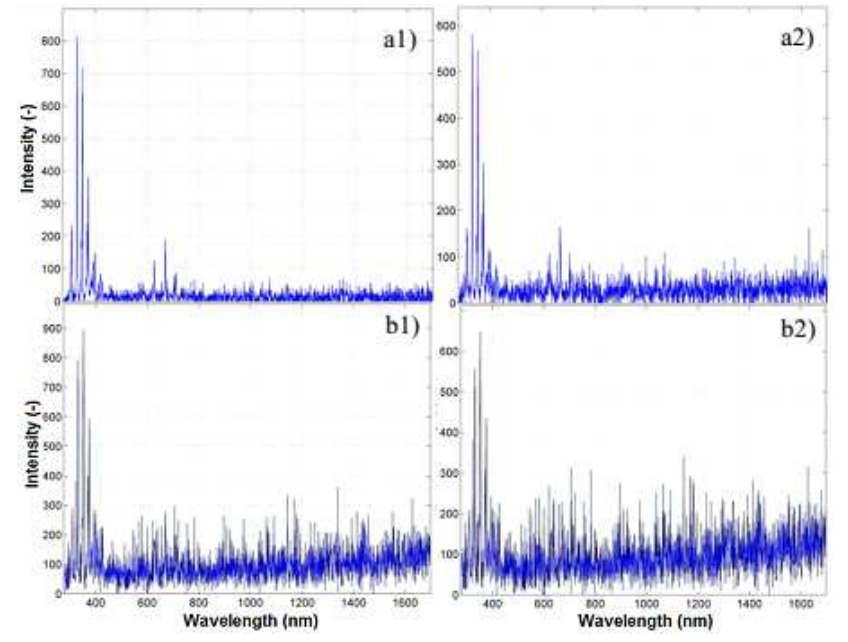

Fig. 2. Spectrum of optical radiation emitted by PDs, when increasing (1) and decreasing (2) values of supply voltage, designated at the value of $U=0.99 U_{\mathrm{b}}$, for: (a1), (a2) bushing insulator - model I; (b1), (b2) bushing insulator - model II.

In order to determine the effect of the supply voltage control method on the obtained measurement results of optical radiation emitted by PDs, characteristics were determined, which are presented in Fig. 3a and $b$. They represent dependences of the intensity on the value of voltage and are plotted separately, when increased or decreased. Graphs were designated for the wavelength equal to $333 \mathrm{~nm}$, for which, regardless the system for modeling the insulator, the maximum intensity value was obtained, either increasing and decreasing the supply voltage. In an analogous manner, characteristics were designated, which were calculated for other dominant wavelengths in the spectra, with the following values of: $352,375,626,671$, and $711 \mathrm{~nm}$. It should be noted that for each of them, the same nature of the examined dependences was obtained.

Figures 4 presents graphs of changes in the intensity of optical radiation, designated for comparative purposes, when increasing (a1, b1) and decreasing (a2, b2) values of supply voltage in systems modeling bushing insulators.

To highlight the structures with dominant values of

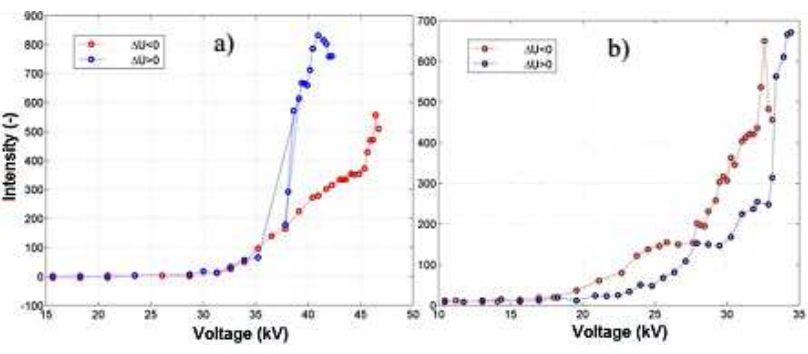

Fig. 3. Change in the intensity of optical radiation emitted by PDs, when increasing $(\Delta U>0)$ and decreasing $(\Delta U<0)$ the supply voltage, at $\lambda=333 \mathrm{~nm}$, for insulator of: (a) bushing type - model I; (b) bushing type - model II.
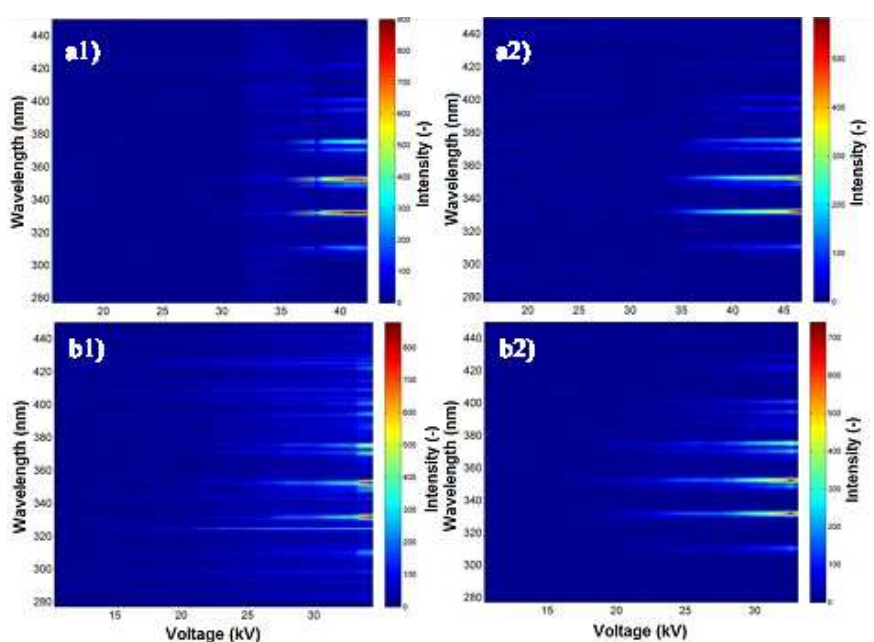

Fig. 4. Comparison of changes in the intensity of optical radiation emitted by PDs at voltage raise (a) and drop (b), determined for wavelengths in the range 270 $450 \mathrm{~nm}$, for an insulator of: (a1), (a2) bushing type model I, (b1), (b2) bushing type - model II.

intensity and thus, to simplify the interpretation of the obtained dependences, characteristics were presented for the range of wavelengths, circumscribed from $270 \mathrm{~nm}$ to $450 \mathrm{~nm}$. The obtained dependences were described using a colour scale in the form of individual strips of colours, corresponding to specific values of the registered intensity emitted by optical radiation.

Figures 5 and 6 present graphs illustrating dependences between intensity fluctuations of optical radiation emitted by PDs in the examined systems modeling bushing insulators and changes in supply voltage values. Characteristics presented in Fig. 5 refer to the case, when the supply voltage increases, while graphs in Fig. 6 were designated, when it is decreasing.

For each of the analysed cases, values derived directly from measurements (single blue dots) and dependences obtained for two selected models: eAU (black line) and AUB (red line) were presented. Characteristics illustrated in Figs. 5 and 6 were designated at the wavelength 


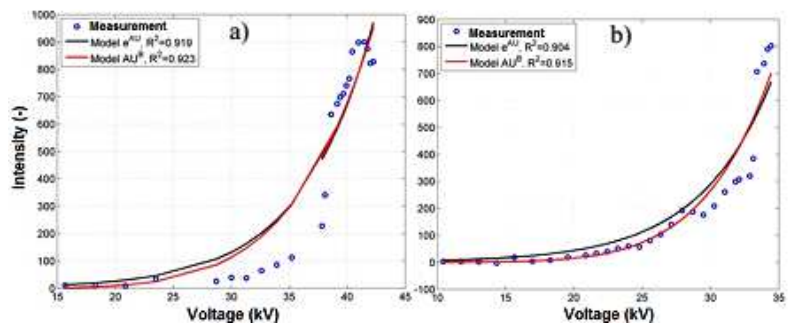

Fig. 5. Change in intensity of optical radiation emitted by PDs with increasing the supply voltage, obtained during measurements and modeled for $\lambda=333 \mathrm{~nm}$, for: (a) bushing insulator - model I, (b) bushing insulator - model II.

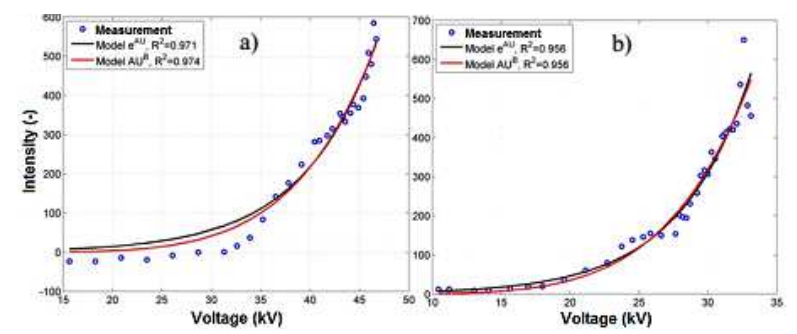

Fig. 6. Change in intensity of optical radiation emitted by PDs with increase of the supply voltage, obtained during measurements and modeled for $\lambda=333 \mathrm{~nm}$, for: (a) bushing insulator - model I, (b) bushing insulator - model II.

of $333 \mathrm{~nm}$, for which the maximum intensity value of the registered optical radiation was obtained.

Tables I, II summarize values of coefficients derived for selected models and their corresponding values of coefficient of determination $\left(R^{2}\right)$. Designated values were presented separately for each of the examined models of insulators, considering the dominant wavelengths of: 333, 352 , and $375 \mathrm{~nm}$, individually for supply voltage raise and drop.
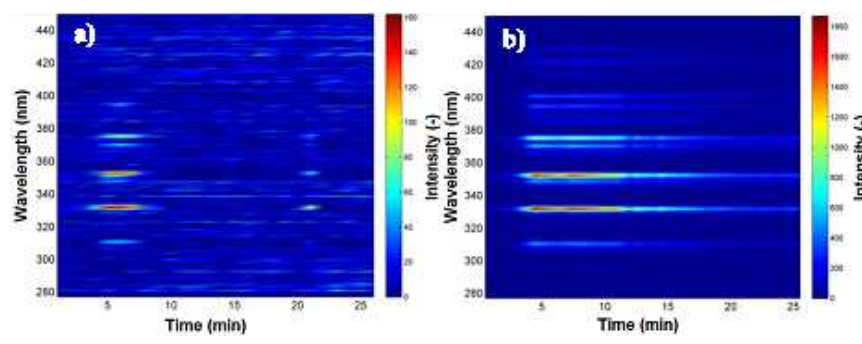

Fig. 7. Change in intensity of optical radiation emitted by PDs as a function of time at constant value of supply voltage $U=0.99 U_{\mathrm{b}}$, for wavelength in the range $270-$ $450 \mathrm{~nm}$, for insulator of: (a) bushing type - model I, (b) bushing type - model II.

Figure 7 shows the dependence of intensity of optical radiation as a function of time for $\mathrm{PD}$ generation on bushing insulator modelling systems. For more accurate
TABLE I

Values of coefficients for the designated models and $R^{2}$ coefficient of determination calculated for two models describing the dependence between intensity in optical radiation emitted by PDs and the change (raise or drop) of the supply voltage, for dominant wavelengths, for bushing insulator model I.

\begin{tabular}{c|c|c|c|c|c}
\hline \hline \multirow{2}{*}{$\begin{array}{c}\text { The spectrum } \\
\text { of dominant } \\
\text { wavelengths } \\
\text { [nm] }\end{array}$} & \multicolumn{5}{|c}{ Model } \\
\cline { 2 - 6 } & $A$ & $\mathrm{e}^{A U}$ & $A$ & $B$ & $R^{2}$ \\
\hline \multicolumn{6}{c}{ Voltage raise } \\
\hline 333 & 0.135 & 0.971 & $1.13 \times 10^{-7}$ & 5.79 & 0.974 \\
352 & 0.127 & 0.963 & $1.58 \times 10^{-7}$ & 5.61 & 0.966 \\
375 & 0.093 & 0.886 & $4.04 \times 10^{-9}$ & 6.19 & 0.912 \\
\hline \multicolumn{6}{c}{ Voltage drop } \\
\hline 333 & 0.144 & 0.921 & $2.62 \times 10^{-8}$ & 6.29 & 0.929 \\
352 & 0.155 & 0.909 & $2 \times 10^{-8}$ & 6.48 & 0.916 \\
375 & 0.123 & 0.896 & $2.27 \times 10^{-8}$ & 6.10 & 0.905 \\
\end{tabular}

TABLE II

Values of coefficients for the designated models and $R^{2}$ coefficient of determination calculated for two models describing the dependence between intensity in optical radiation emitted by PDs and the change (raise or drop) of the supply voltage, for dominant wavelengths, for bushing insulator model II.

\begin{tabular}{c|c|c|c|c|c}
\hline \hline \multirow{2}{*}{$\begin{array}{c}\text { The spectrum } \\
\text { of dominant } \\
\text { wavelengths } \\
\text { [nm] }\end{array}$} & \multicolumn{5}{c}{ Model } \\
\cline { 2 - 6 } & $A$ & $\mathrm{e}^{A U}$ & \multicolumn{3}{c}{$A U^{B}$} \\
\hline \multicolumn{6}{c}{ Voltage raise } \\
\hline 333 & 0.189 & 0.904 & $1.06 \times 10^{-8}$ & 7.04 & 0.915 \\
352 & 0.186 & 0.914 & $1.14 \times 10^{-8}$ & 6.99 & 0.926 \\
375 & 0.170 & 0.936 & $2.35 \times 10^{-5}$ & 4.65 & 0.923 \\
\hline \multicolumn{6}{c}{ Voltage drop } \\
\hline 333 & 0.195 & 0.962 & $7.49 \times 10^{-5}$ & 5.87 & 0.963 \\
352 & 0.192 & 0.967 & $1.71 \times 10^{-6}$ & 5.60 & 0.968 \\
375 & 0.160 & 0.933 & $1.25 \times 10^{-5}$ & 4.73 & 0.933
\end{tabular}

analysis of changes occurring in the obtained structures, graphs were developed for restricted to dominant wavelengths in the spectrum within the range of 270-450 nm. Recording time was $25 \mathrm{~min}$. Intensity values are illustrated by a bar with palette of colours.

In order to determine the effect of time, during which PDs are generated, on the shape of designated spectra of optical radiation, graphs illustrating the dependence between intensity and emitted wavelengths were summarized in Fig. 8 for comparison of both systems modeling bushing insulators. Spectra were designated for the time point of $15 \mathrm{~min}$ and shown in the range of dominant wavelengths from $270 \mathrm{~nm}$ to $450 \mathrm{~nm}$.

\section{Conclusion}

Application of the spectrophotometer allowed for registration and analysis of optical radiation within a wide 


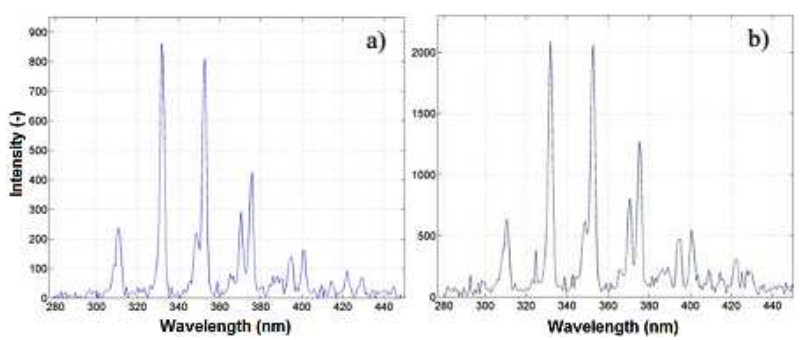

Fig. 8. Spectrum of optical radiation emitted by PDs as a function of time at constant value of supply voltage $U=0.99 U_{\mathrm{b}}$, for a wave in the range $270-450 \mathrm{~nm}$, at the time point of $15 \mathrm{~min}$, for: (a) bushing insulator model I, (b) bushing insulator — model II.

spectral band, respectively in the range of $270 \mathrm{~nm}$ to $1700 \mathrm{~nm}$, which covered the range of: ultraviolet, visible and infrared radiation. The obtained results indicate that the largest energy contribution is in the spectra of the registered electromagnetic waves, emitted by the modeled PDs, UV-A radiation within the range of 315$400 \mathrm{~nm}$. The optical spectroscopy method, applied in laboratory tests, allows for detection of partial discharges generated in the proposed bushing insulator modeling system; however, this applies to supply voltages exceeding $0.7 U_{\mathrm{b}}$.

In particular, based on the performed measurements and tests on intensity of optical radiation emitted by PD generated in bushing insulator models, the results of which are presented in this paper, it can be stated that:

- Designated spectra have two local intensity extrema that occur in the ranges of: approximately 280-430 $\mathrm{nm}$ and about 500-720 nm. Radiation intensity in the ultraviolet range, particularly in UV-A (the range was adopted based on ISO 21348:2007(E) Space environment natural and artificial - Process for determining solar irradiances), that means in the range between $315 \mathrm{~nm}$ and $400 \mathrm{~nm}$, is much higher than in the visible range $380-780 \mathrm{~nm}$. Radiation intensity values maintained within the UV-A range are on average over three times higher than the corresponding ones, registered in the visible range. For wavelengths exceeding $720 \mathrm{~nm}$, the calculated spectra have flat waveforms, without visible extremes. This indicates a relatively small infrared radiation (abbreviation IR) in the spectrum of optical radiation generated by the modeled PDs. In particular this applies to near infrared (IR-A) radiation, that is in the range between $780 \mathrm{~nm}$ and $1400 \mathrm{~nm}$.

- Within the designated spectra, three dominant wavelengths occur that are equal to, respectively: 333,352 , and $375 \mathrm{~nm}$, the intensity of which is decisively the largest in the whole analysed range of the emitted optical radiation. The size of inten- sity corresponding to these wavelengths, depends on a method for controlling (reducing and increasing) the power supply voltage, and also depends on the model of insulator applied. It can be noted that for bushing insulator (model I), the highest value of intensity occurs for the wavelength of $333 \mathrm{~nm}$ and the lowest for the length of $375 \mathrm{~nm}$. Whereas, for bushing insulator (model II), the highest value of intensity is for the wavelength of $352 \mathrm{~nm}$ and the lowest for the length of $375 \mathrm{~nm}$. Besides the mentioned UV dominant optical wavelengths, several lengths can be also distinguished that are of: 312 , $389,394,401$, and $422 \mathrm{~nm}$, respectively, which are characterised by increased intensity values. They occur in both tested models of insulators, regardless the method for controlling of power supply voltage. At the same time, intensities corresponding to these wavelengths are differentiated, depending on the tested model, and significantly lower than the intensity of the dominant wavelengths in the designated optical radiation spectra.

- Within the visible range, three wavelengths can be identified, that is: 626,671 , and $711 \mathrm{~nm}$, for which the radiation intensity values are in this range the highest. While the values obtained for the three dominant wavelengths, mentioned above, are on average more than three times lower in the UV-A range.

- Obtained waveforms of the spectrum of optical radiation are independent of the voltage value of PD generation. This applies to the voltage range from the ignition of discharges (approximately $0.5 U_{\mathrm{b}}$ ) to the value equal to $0.99 U_{\mathrm{b}}$. For minimum voltage values (less than $0.7 U_{\mathrm{b}}$ ), the intensity of radiation emitted by PDs is significantly lower and is characterized by relatively low stability, and the examined phenomena are characterized by strong stochasticity threshold.

- No impact of changes in the value of power supply voltage on shape of the spectral waveforms obtained and dominant wavelengths was observed; and this applies to both, the increase and decrease of the value.

- Spectral waveforms of intensity for the optical radiation, obtained from measurements, for selected relative voltage values, were modeled using a series of Gaussian functions. Mean value of particular components is determined by the wavelength of radiation component. The models applied are characterized by high degree of correlation in relation to the coefficient of determination $R^{2}$, which is above 0.7 .

- Shape of the calculated optical radiation spectra does not change over time (registration time $25 \mathrm{~min}$ ) for a given and constant voltage value of 
PD generation. This proposal applies as well to the dominant wavelength in the registered spectra and for the both examined systems modeling bushing insulators. The observed changes concern absolute values of intensity size that is emitted by optical radiation for the distinguished wavelengths, which indicates fluctuating and largely stochastic nature of the generated PDs.

\section{Acknowledgments}

The work was supported by the National Centre for Research and Development within the LIDER program.

\section{References}

[1] A.H. El-Hag, Y.A. Saker, I.Y. Shurrab, IEEE Trans. Power Deliv. 26, 1288 (2011).

[2] M. Zdanowski, S. Wolny, D. Zmarzły, T. Boczar, J. Electrostat. 65, 239 (2007).

[3] S. Borucki, T. Boczar, A. Cichoń, Arch. Acoust. 36 , 49 (2011).

[4] E. Lindell, T. Bengtsson, J. Blennow, S.M. Gubanski, IEEE Trans. DEI 18, 246 (2011).

[5] L. Junhao, H. Quanwei, Z. Xuefeng, Y. Xiu, Y. Yongfen, Y. Yanming, IEEE Trans. Power Deliv. 26, 538 (2011).

[6] D. Wotzka, T. Boczar, D. Zmarzły, Acta Phys. Pol. A 116, 428 (2009).

[7] A. Cavallini, G.C. Montanari, M. Tozzi, X. Chen, IEEE Trans. DEI 18, 275 (2011).
[8] Y. Kikuchi, T. Murata, N. Fukumoto, M. Nagata, Y. Wakimoto, T. Yoshimitsu, IEEE Trans. DEIS 17, 839 (2010).

[9] S.E.U. Lima, O. Frazao, R.G. Farias, F.M. Arauějo, L.A. Ferreira, J.L. Santos, V. Miranda, IEEE Trans. Power Deliv. 25, 2526 (2010).

[10] S. Rudd, S.D.J. Mcarthur, M.D. Judd, IEEE Trans. DEI 17, 149 (2010).

[11] M. Pompili, R. Bartnikas, IEEE Trans. DEI 19, 1476 (2012).

[12] S. Biswas, C. Koley, B. Chatterjee, S. Chakravorti, IEEE Trans. DEI 19, 18 (2012).

[13] W. Kai, P. Cheng, M. Yongpeng, S. Changhao, G. Minggang, Q. Kai, L. Hongyu, IEEE Trans. DEI 18, 1651 (2011).

[14] T. Boczar, D. Zmarzły, Brit. Instit. Non-Destruct. Test. 45, 488 (2003).

[15] R. Bartnikas, IEEE Trans. DEI 9, 763 (2002).

[16] D. Wotzka, A. Cichoń, T. Boczar, Arch. Acoust. 37, 19 (2012).

[17] Y. Cheng, C. Li, X. Huang, IEEE Trans. Power Deliv. 23, 945 (2008).

[18] S. Gubański, A. Dernfalk, J. Andersson, H. Hillborg, IEEE Trans. DEI 15, 1065 (2007).

[19] W. He, T. Chen, X. Liu, F. Yang, D. Yao, D. Xiong, Automat. Electr. Power Syst. 10, 69 (2006).

[20] T. Kikuchi, S. Nishimura, M. Nagano, K. Izumi, Y. Kubota, M. Sakata, IEEE Trans. DEI 6, 548 (1999).

[21] X. Meng, W. Cao, High Voltage Eng. 6, 42 (2006).

[22] T. Boczar, D. Zmarzły, IEEE Trans. DEI 13, 632 (2006). 